\title{
Perfil de risco cardiovascular \\ e autoavaliação da saúde no Brasil: estudo de base populacional
}

\author{
Janaina Caldeira Pereira, ${ }^{1}$ Sandhi Maria Barreto ${ }^{1}$ \\ e Valéria Maria de Azeredo Passos ${ }^{1}$
}

Como citar Pereira JC, Barreto SM, Passos VMA. Perfil de risco cardiovascular e autoavaliação da saúde no Brasil: estudo de base populacional. Rev Panam Salud Publica. 2009;25(6):491-8.

RESUMO Objetivos. Estimar a prevalência e a aglomeração de fatores de risco cardiovascular (hipertensão, tabagismo, inatividade física, uso excessivo de álcool, dieta inadequada e obesidade central) e investigar a sua associação com autoavaliação da saúde após ajuste por características sociodemográficas e diagnóstico médico de diabetes e doença isquêmica do coração.

Métodos. Participaram 9211 brasileiros de 30 a 69 anos, residentes em 16 capitais, incluídos no Inquérito Domiciliar sobre Comportamentos de Risco e Morbidade Referida de Doenças e Agravos Não Transmissíveis, 2002-2003, um estudo transversal de base populacional realizado pelo Ministério da Saúde. A variável resposta foi o escore de aglomeração de fatores de risco cardiovascular, variando de zero a 3: nenhuma exposição ou exposição a 1, 2 e 3 ou mais fatores de risco. Os dados foram analisados por regressão logística multinomial, tendo como referência a categoria zero de fatores de risco cardiovascular, com e sem hipertensão.

Resultados. A prevalência dos fatores de risco, exceto uso de álcool, foi superior a $25 \%$. A frequência de 2 ou mais fatores de risco foi de 47,49\%; 17,91\% dos participantes não apresentavam nenhum fator. A aglomeração de fatores de risco foi inversamente associada com renda e escolaridade e foi maior nas idades entre 50 e 59 anos, sem diferenças significativas entre os sexos. Uma pior autoavaliação de saúde foi mais frequente em participantes com mais fatores de risco. A inclusão da hipertensão no escore representou piora no perfil de risco com o aumento da idade e associação significativa com doença isquêmica do coração e diabetes referidos. Conclusões. A elevada prevalência de fatores de risco cardiovascular em adultos brasileiros reforça a importância de politicas voltadas para a redução desses fatores. A associação entre pior percepção da saúde e maior número de fatores de risco cardiovascular chama a atenção para a importância, a abrangência e a complexidade desse marcador subjetivo de saúde em inquéritos populacionais e sugere que a autopercepção de uma saúde ruim não é suficiente para uma atitude saudável.

Palavras-chave Doenças cardiovasculares, fatores de risco, auto-imagem, Brasil.

No Brasil, as doenças cardiovasculares (DCV) representam aproximadamente $30 \%$ de todos os óbitos. Desses, cerca de $50 \%$ acometem adultos entre 30 e 69

\footnotetext{
Universidade Federal de Minas Gerais (UFMG), Faculdade de Medicina. Enviar correspondência a Janaina Caldeira Pereira no seguinte endereço: Rua Itapemirim 176/404, CEP 30240-000, Belo Horizonte, MG, Brasil. E-mail: janacgodoy@yahoo. com.br
}

anos, em plena fase produtiva (1). A mortalidade prematura em adultos e as incapacidades resultantes, parciais ou totais, têm importantes repercussões na qualidade de vida e na rede social dos acometidos, além do grande impacto nos sistemas de saúde.

Apesar da gravidade das DCV e do aumento de sua incidência com o avanço da idade, grande parte dessas doenças poderia ser evitada. Como as doenças crônicas não transmissíveis mais frequentes - DCV, diabetes e câncer compartilham vários fatores de risco, a Organização Mundial da Saúde (OMS) propõe uma abordagem de prevenção e controle integrados, em todas as idades, baseada na redução dos seguintes fato- 
res: hipertensão arterial, tabagismo, uso de álcool, inatividade física, dieta inadequada, obesidade e hipercolesterolemia (2). Estudos mostram que esses fatores tendem a ocorrer simultaneamente com frequência maior do que aquela esperada estritamente pela casualidade. Além disso, a agregação de fatores de risco cardiovascular está associada a um risco maior do que o esperado pela soma dos riscos individuais de cada fator (3). As estimativas do efeito da aglomeração desses fatores consideram que muitos são inter-relacionados e participam também como intermediários em uma mesma cadeia causal (4).

Muitas das variações observadas no perfil individual ou populacional de saúde cardiovascular podem ser compreendidas por meio do estudo da aglomeração de fatores de risco, já que o comportamento desses fatores varia segundo características sociodemográficas, étnicas, geográficas e também quanto à presença de doenças e a percepção da própria saúde (3, 5-8). Apesar da importância desse tema, o volume de informações de base populacional sobre a aglomeração de fatores de risco cardiovascular (FRCV) na população adulta brasileira ainda é restrito (9-11). Pouco se sabe também sobre a relação entre a aglomeração de FRCV e a autoavaliação da saúde entre adultos brasileiros. Nos Estados Unidos e na Suécia, um estudo recente mostrou uma forte associação entre a carga de fatores de risco cardiovascular e a autoavaliação da saúde em adultos (12).

Dessa forma, o objetivo do presente estudo foi analisar dados do Inquérito Domiciliar sobre Comportamentos de Risco e Morbidade Referida de Doenças e Agravos Não Transmissíveis, do Ministério da Saúde, a fim de traçar o perfil da aglomeração de FRCV em adultos de 30 a 69 anos no Brasil e investigar a sua associação com características sociodemográficas, auto-avaliação da saúde e relato de diagnóstico médico de diabetes e doença isquêmica do coração (DIC).

\section{MATERIAIS E MÉTODOS}

A população do estudo é composta por participantes do Inquérito Domiciliar sobre Comportamentos de Risco e Morbidade Referida de Doenças e Agravos Não Transmissíveis realizado pelo Instituto Nacional de Câncer (INCA) e pela Secretaria de Vigilância à Saúde do Ministério da Saúde no período de 2002 a
2003. Trata-se de um estudo transversal de base populacional com 23457 participantes de 15 ou mais anos de idade, residentes em 16 capitais: Aracaju, Belém, Belo Horizonte, Brasília, Campo Grande, Curitiba, Florianópolis, Fortaleza, João Pessoa, Manaus, Natal, Porto Alegre, Recife, Rio de Janeiro, São Paulo e Vitória. A amostra autoponderada foi selecionada em dois estágios. Outros detalhes sobre a metodologia e a coleta da amostra encontram-se na publicação oficial do Inquérito (13). O banco de dados para análise de dados secundários selecionados foi obtido junto à Secretaria de Vigilância em Saúde e ao INCA por uma das autoras (SMB). Duas autoras (SMB e VMAP) participaram da elaboração de capítulos da publicação oficial do inquérito.

O presente estudo incluiu 9211 adultos $(39,26 \%$ da amostra total) de ambos os sexos, com idade entre 30 e 69 anos. $\mathrm{O}$ limite etário inferior foi definido em função do aumento da incidência de doenças crônicas a partir dos 30 anos (14). Foram excluídos idosos com 69 anos ou mais, que não foram submetidos à investigação sobre atividade física.

Quatro grupos de variáveis foram analisados: 1) características sociodemográficas: idade (30 a 39, 40 a 49, 50 a 59 e 60 a 69 anos), sexo, escolaridade ( 0 a 3, 4 a 7, 8 a 11,12 ou mais anos) e renda familiar (até 3,3 a $<5, \geq 5$ a $<10$ e $\geq 10$ salários mínimos); 2) fatores de risco cardiovascular modificáveis (hipertensão arterial sistêmica, tabagismo, consumo de álcool considerado de risco, inatividade física, dieta inadequada e obesidade central); 3) morbidade referida (doenças): relato de diagnóstico médico de doença isquêmica do coração e diabetes; e 4) percepção da própria saúde (excelente/muito boa, boa, regular ou ruim).

A variável resposta foi a aglomeração de FRCV. Para essa análise, foram incluídos os fatores de risco com percentuais de resposta superiores a $85 \%$ na amostra selecionada: 91,8\% ( $n=8457)$ para dieta; $91,2 \%(n=8396)$ para tabagismo; $90,0 \%$ $(n=8290)$ para inatividade física; $89,4 \%$ $(n=8240)$ para consumo de álcool; $87,6 \%(n=8070)$ para hipertensão; e $86,8 \%(n=7996)$ para obesidade central. Foi criado um escore de aglomeração para retratar a presença de qualquer combinação entre esses fatores. O escore variou de zero a 3 (zero = nenhuma exposição, 1 = exposição a um fator; 2 = exposição a dois fatores; e 3 = exposição a três ou mais fatores de risco).
Para avaliar se a dieta era inadequada, foram utilizadas informações sobre a frequência semanal do consumo de frutas e/ou legumes e/ou hortaliças. Considerou-se que havia risco quando o entrevistado informou que a ingestão desses alimentos era inferior a 5 vezes por semana (13).

Quanto ao hábito de fumar, os indivíduos foram classificados em três categorias: fumantes (fumaram pelo menos 100 cigarros na vida e fumavam na ocasião do inquérito), ex-fumantes (fumaram pelo menos 100 cigarros na vida, mas não fumavam na ocasião do inquérito) e não fumantes (15). O nível de atividade física foi aferido por um questionário (International Physical Activity Questionnaire, IPAQ) em sua versão reduzida. O questionário é recomendado pela OMS para avaliação da atividade física em adultos de 15 a 69 anos (16). O instrumento contém perguntas relacionadas à frequência (dias por semana), duração (tempo por dia) e intensidade (leve, moderada e vigorosa) da atividade física nos últimos 7 dias. Os indivíduos são classificados como muito ativos, ativos, irregularmente ativos e sedentários, de acordo com o escore obtido. Foram considerados grupos de risco aqueles classificados como irregularmente ativos ou sedentários.

O consumo alcoólico de risco foi identificado entre homens que relataram consumir mais de duas doses de bebida alcoólica por dia e entre mulheres que relataram o consumo de mais de uma dose de bebida alcoólica por dia nos últimos 30 dias. Considerou-se como dose uma meia garrafa ou uma lata de cerveja, um cálice de vinho ou um drinque de bebida destilada (aguardente, uísque, etc.) (15). Foi considerada como obesidade central a medida de cintura maior do que $88 \mathrm{~cm}$ nas mulheres e $102 \mathrm{~cm}$ nos homens. O ponto de corte utilizado baseouse nas recomendações da OMS (17).

A presença de hipertensão arterial (HAS) foi determinada pela resposta positiva à pergunta "algum médico, enfermeiro ou agente comunitário de saúde já lhe disse que o(a) Sr.(a.) tem pressão alta?". Os indivíduos que referiram DIC responderam positivamente à seguinte questão: "algum médico já lhe disse que o(a) Sr.(a.) tem ou teve ataque do coração ou enfarte ou angina ou doença das coronárias?". Os diabéticos foram classificados em dois grupos: indivíduos que referiram já ter realizado exame para medir açúcar no sangue nos últimos 2 
TABELA 1. Prevalências de fatores de risco cardiovascular modificáveis por faixa etária, Brasil, 2002 a 2003

\begin{tabular}{|c|c|c|c|c|c|c|}
\hline \multirow[b]{2}{*}{ Fator de risco } & \multicolumn{6}{|c|}{ Faixa etária (anos) } \\
\hline & $\begin{array}{c}30 \text { a } 39 \\
\text { Prevalência }^{a} \text { (IC95\%) }\end{array}$ & $\begin{array}{c}40 \text { a } 49 \\
\text { Prevalência }{ }^{a} \text { (IC95\%) }\end{array}$ & $\begin{array}{c}50 \text { a } 59 \\
\text { Prevalência }^{a} \text { (IC95\%) }\end{array}$ & $\begin{array}{c}60 \text { a } 69 \\
\text { Prevalência }{ }^{a} \text { (IC95\%) }\end{array}$ & Total & $\chi^{2} / P^{\mathrm{b}}$ \\
\hline Dieta inadequada & $66,91(64,33$ a 69,40$)$ & $70,66(67,70$ a 73,45$)$ & $70,69(66,93$ a 74,20$)$ & $71,89(67,57$ a 75,85$)$ & $69,44(67,76$ a 71,07$)$ & $15,08 / 0,105$ \\
\hline Inatividade física & $40,73(38,06$ a 43,45$)$ & $38,97(35,98$ a 42,04$)$ & $41,06(37,37$ a 44,86$)$ & $41,39(36,81$ a 46,12$)$ & $40,33(38,41$ a 42,29$)$ & $3,01 / 0,70$ \\
\hline Obesidade central & $17,74(15,58$ a 20,12$)$ & $27,47(24,99$ a 30,11$)$ & $41,18(38,06$ a 44,37$)$ & $37,42(33,26$ a 41,77$)$ & $27,99(26,47$ a 29,56$)$ & $337,21 / 0,00$ \\
\hline Hipertensão & $12,89(11,22$ a 14,77$)$ & $25,79(23,14$ a 28,64$)$ & $41,73(38,43$ a 45,11$)$ & $47,33(42,57$ a 52,14$)$ & $27,16(25,71$ a 28,66$)$ & $680,44 / 0,00$ \\
\hline \multicolumn{7}{|l|}{ Fumo } \\
\hline Fumantes & $18,32(16,38$ a 20,44$)$ & $28,87(26,22$ a 31,67$)$ & $30,93(27,79$ a 34,25$)$ & $30,60(26,74$ a 34,76$)$ & $25,66(24,21$ a 27,18$)$ & $204,75 / 0,00$ \\
\hline Ex-fumantes & $21,99(19,81$ a 24,34$)$ & $26,71(24,31$ a 29,26$)$ & $21,96(19,31$ a 24,87$)$ & $17,64(14,21$ a 21,67$)$ & $22,94(21,63$ a 24,31$)$ & $204,75 / 0,00$ \\
\hline Álcool & $7,97(6,82$ a 9,29$)$ & $9,76(8,12$ a 11,7$)$ & $7,11(5,60$ a 8,99$)$ & $4,31(2,51$ a 7,29$)$ & $7,91(7,06$ a 8,85$)$ & $30,74 / 0,004$ \\
\hline
\end{tabular}

Fonte: Inquérito Domiciliar sobre Comportamentos de Risco e Morbidade Referida de Doenças e Agravos Não Transmissíveis, Comprev/INCA/Ministério da Saúde (13).

${ }^{a}$ As porcentagens levam em consideração o peso amostral dos indivíduos.

${ }^{\text {b }}$ Qui-quadrado para as variáveis apresentadas e grupos de idades.

anos; e indivíduos com diagnóstico de diabetes feito por um médico ("algum médico já lhe disse que o(a) Sr.(a.) tem diabetes?"). Para a autoavaliação da saúde, os entrevistados tomaram como base a saúde de pessoas da sua faixa etária e definiram comparativamente o seu próprio estado de saúde como excelente ou muito bom, bom, regular e ruim.

Foram feitas distribuições de todas as variáveis por faixa etária e sexo, usando medidas de proporção pela estimativa do qui-quadrado $\left(\chi^{2}\right)$ de Pearson e seu intervalo de confiança de $95 \%$ (IC95\%). O escore de aglomeração de FRCV foi analisado com e sem inclusão da variável hipertensão. A análise univariada verificou a associação do escore com variáveis sociodemográficas, doenças (diabetes e DIC) e percepção da própria saúde. As associações que apresentaram valor de $P<0,20$ foram incluídas no modelo multivariado. Nas análises que não incluíram a variável hipertensão no escore, o modelo foi ajustado também por esta variável. Considerou-se significativa a associação cujo valor de $P$ foi menor do que 0,05 . A magnitude das associações e respectivos IC95\% foram estimados pela regressão logística multinomial, tendo como referência a categoria do escore "nenhuma exposição". Foi utilizado o programa STATA 9.0, que dispõe de procedimentos para análise de inquéritos amostrais complexos. Esses procedimentos permitem incorporar os pesos distintos das observações que influenciam as estimativas pontuais de parâmetros da população total.

$\mathrm{O}$ inquérito foi aprovado pelo Comitê de Ética em Pesquisa do INCA e pelo Comitê Nacional de Ética em Pesquisa, e conduzido de forma a proteger a privacidade dos indivíduos, garantindo participação anônima e voluntária.

TABELA 2. Distribuição de adultos de 30 a 69 anos de acordo com aglomeração de fatores de risco cardiovascular modificáveis, Brasil, 2002 a 2003

\begin{tabular}{lcc}
\hline $\begin{array}{c}\text { Número de } \\
\text { fatores de risco }\end{array}$ & $\begin{array}{c}\text { Homens }(n=3104) \\
\%(I C 95 \%)^{\mathrm{b}, \mathrm{c}}\end{array}$ & $\begin{array}{c}\text { Mulheres }(n=4364) \\
\%(I C 95 \%)^{\mathrm{b}, \mathrm{c}}\end{array}$ \\
\hline Zero & $19,49(17,57$ a 21,57$)$ & $16,78(15,90$ a 18,50) \\
1 & $34,65(32,44$ a 36,93) & $34,02(32,02$ a 36,06) \\
2 & $25,61(23,44$ a 27,91) & $28,83(26,82$ a 30,92) \\
$\geq 3$ & $20,24(18,27$ a 22,37) & $20,37(18,64$ a 22,22) \\
\hline
\end{tabular}

Fonte: Inquérito Domiciliar sobre Comportamentos de Risco e Morbidade Referida de Doenças e Agravos Não Transmissíveis, Comprev/INCA/Ministério da Saúde (13).

a Escore de aglomeração de fatores de risco cardiovascular definido como a soma de qualquer combinação entre os seguintes fatores: hipertensão, tabagismo, dieta inadequada, uso excessivo de álcool, inatividade física e obesidade central.

${ }^{\mathrm{b}}$ As proporções levam em consideração o peso amostral dos indivíduos.

${ }^{c}$ Comparação entre homens e mulheres: $\chi^{2}=16,08 ; P=0,09$.

\section{RESULTADOS}

Dos 9211 adultos participantes, 5042 $(54,7 \%)$ eram mulheres e $4169(45,3 \%)$ eram homens. A média de idade foi de 45,08 anos $( \pm 0,14)$ e $67,71 \%$ tinham idade inferior a 50 anos. A escolaridade média foi igual a 8,1 anos (IC95\%: 7,8 a 8,4), reduzindo-se significativamente com o avançar da idade. A renda familiar foi inferior a 5 salários mínimos para 50,49\% (IC95\%: 47,6 a 53,4) dos entrevistados.

As prevalências dos FRCV modificáveis por faixa etária e os respectivos intervalos de confiança são apresentados na tabela 1 . Os fatores mais e menos prevalentes foram a dieta inadequada $(69,44 \%)$ e o consumo de álcool $(7,91 \%)$, respectivamente. Não foram encontradas associações significativas entre idade e dieta inadequada ou inatividade física. $\mathrm{O}$ aumento da prevalência com o avançar da idade foi particularmente evidente para a hipertensão.

$\mathrm{Na}$ análise separada por sexo, foram observados, com o aumento da idade, aumentos significativos nas prevalências de HAS e obesidade central em ambos os sexos, e de tabagismo entre os homens. Não houve associação entre idade e inatividade física. Dieta inadequada e consumo de álcool apresentaram associação significativa com idade apenas entre mulheres.

A tabela 2 ilustra a distribuição de homens e mulheres de acordo com a aglomeração de fatores de risco. Observa-se grande similaridade entre os dois grupos, evidenciada pela superposição dos intervalos de confiança.

Na figura 1, a distribuição da aglomeração de FRCV por faixa etária é apresentada com e sem inclusão da variável hipertensão na composição do escore. Em toda a população investigada, 17,91\% não relataram qualquer fator de risco modificável, incluindo hipertensão. Essa proporção diminuiu significativamente com avançar da idade.

Participantes com dois ou mais fatores somaram $47,49 \%$ do total investigado. A faixa etária com pior perfil de risco foi a de 50 a 59 anos. A inclusão de HAS no escore representou piora no perfil de risco 
FIGURA 1. Aglomeração de fatores de risco cardiovascular por faixa etária, com e sem inclusão de hipertensão, Brasil, 2002 a 2003

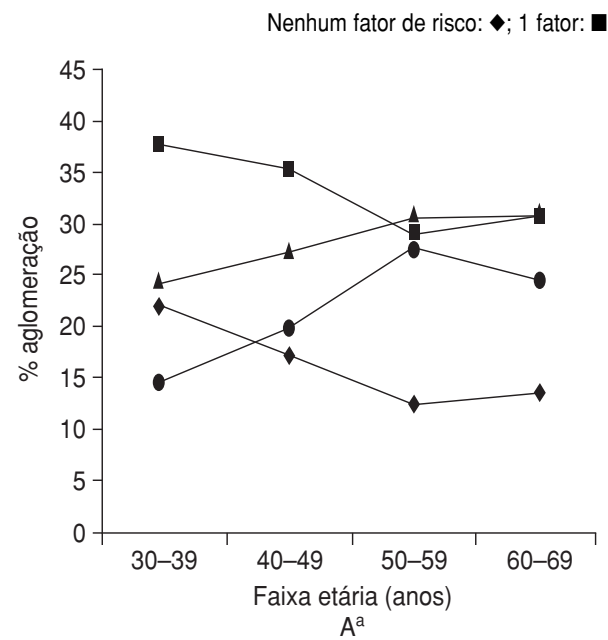

; 2 fatores: $\mathbf{\Lambda} ; 3$ ou mais fatores:

Fonte: Inquérito Domiciliar sobre Comportamentos de Risco e Morbidade Referida de Doenças e Agravos Não Transmissíveis, Comprev/INCA/Ministério da Saúde (13).

a Escore de aglomeração de fatores de risco cardiovascular definido como a soma de qualquer combinação entre os seguintes fatores: hipertensão, tabagismo, dieta inadequada, consumo excessivo de álcool, inatividade física e obesidade central.

${ }^{b}$ Escore de aglomeração de fatores de risco cardiovascular: tabagismo, dieta inadequada, consumo excessivo de álcool, inatividade física e obesidade central. As proporções levam em consideração o peso amostral dos indivíduos.

FIGURA 2. Prevalência de cada fator de risco cardiovascular (FRCV) por categoria do escore de aglomeração

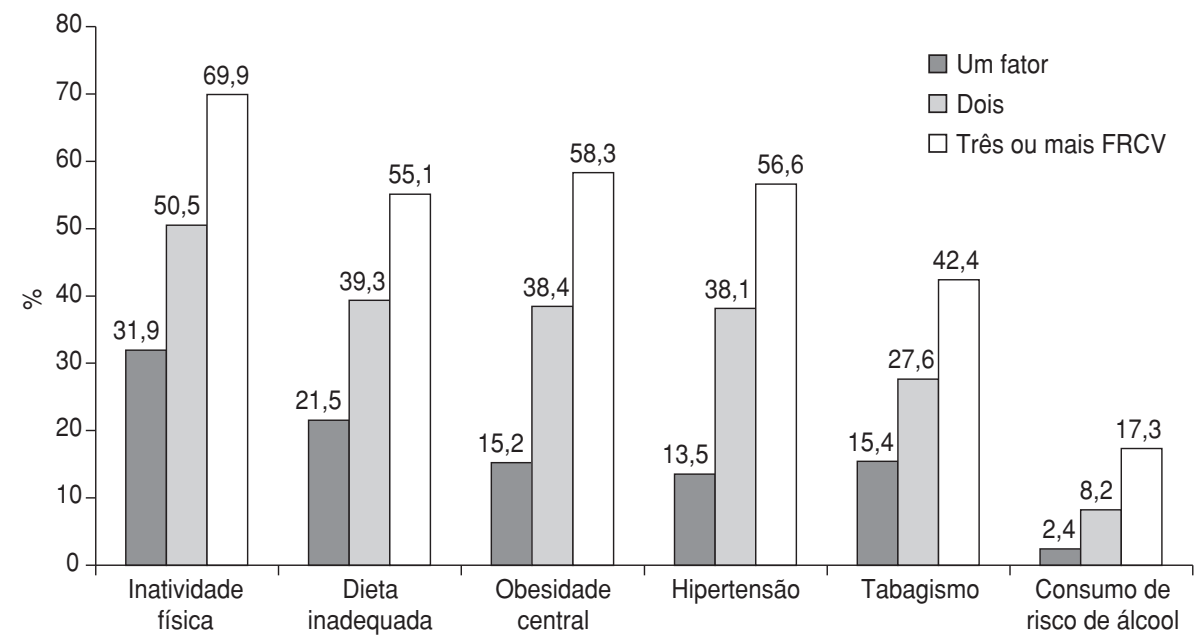

Fonte: Inquérito Domiciliar sobre Comportamentos de Risco e Morbidade Referida de Doenças e Agravos Não Transmissíveis, Comprev/INCA/Ministério da Saúde (13).

com o avançar da idade e redução do número de indivíduos sem fatores de risco em todas as faixas etárias. A aglomeração de 3 fatores de risco em homens de 50 a 59 anos foi, em média, 2,3 vezes maior em relação àqueles na faixa dos 30 a 39 anos (29,12 vs. $\left.12,75 \%, \chi^{2}=108,07 ; P<0,001\right)$.

A figura 2 evidencia a frequência dos fatores dentro de cada categoria do escore de aglomeração. A inatividade física foi o fator mais prevalente nas categorias com 1, 2 e 3 ou mais FRCV. Para os indivíduos que referiram 2 fatores, dieta inadequada, obesidade central e hipertensão apresentaram prevalências próximas como segundo fator de risco. Já entre aqueles que apresentaram 3 ou mais fatores, seguiram-se à inatividade física, por ordem decrescente de prevalências, obesidade central, hipertensão e dieta inadequada.

A tabela 3 mostra os resultados da análise univariada da aglomeração de fatores de risco em função das características so- ciodemográficas, autoavaliação da saúde, diabetes e doença isquêmica do coração. Idade, renda e autoavaliação da saúde apresentaram associações significativas em todas as categorias do escore, com gradiente positivo entre o aumento do número de FRCV e pior avaliação da saúde. Sexo associou-se de forma significativa com a aglomeração de dois FRCV. As probabilidades de referir diabetes e DIC aumentaram de forma significativa com um maior número de fatores de risco.

Foi utilizado um modelo multivariado na análise da associação entre FRCV e idade, sexo, renda e percepção da saúde (tabela 4). Observou-se piora na avaliação de saúde com o aumento do número de fatores de risco. Aqueles que avaliaram sua saúde como ruim, comparados aos que a consideraram excelente ou muito boa, apresentaram uma probabilidade 2,80, 6,13 e 14,60 vezes maior de apresentar 1, 2 e 3 ou mais fatores de risco, respectivamente, tendo como categoria de referência a ausência de FRCV. Não houve mudança na direção da associação entre percepção da saúde e aglomeração de FRCV quando se ajustou o modelo por diabetes e DIC. A pior avaliação da saúde manteve-se associada com maior probabilidade de apresentar FRCV (resultados não apresentados). Observou-se também relação inversa entre aglomeração de FRCV e renda familiar na análise multivariada (tabela 4).

$\mathrm{Na}$ tabela 5, a exclusão de HAS do escore de aglomeração resultou em redução da magnitude das associações. Além de sexo, a variável idade deixou de apresentar significância estatística. $\mathrm{O}$ ajuste por hipertensão, diabetes e DIC manteve as associações com percepção da saúde e renda familiar com mesma direção e pouca variação nas razões de chances para renda.

\section{DISCUSSÃO}

O presente estudo descreveu a distribuição e a aglomeração de FRCV modificáveis na população de 16 capitais do Brasil e investigou sua associação com a autoavaliação da saúde. $O$ inquérito cujos dados foram utilizados (13) forneceu, de modo inédito, informações de abrangência nacional sobre a população urbana. Para países em desenvolvimento que, como o Brasil, dispõem de um serviço de atenção à saúde com cobertura universal, as informações provenientes de autorrelato de diagnóstico médico 
podem ser obtidas com razoável confiabilidade e facilidade, permitindo o estudo das condições de saúde da população e de sua relação com a prevalência de fatores de risco modificáveis. Tal estratégia oferece vantagens importantes, como rapidez na execução e menor custo (18-20).

A elevada prevalência de fatores de risco como dieta inadequada, inatividade física, obesidade central e hipertensão são consistentes com estudos realizados no país e no exterior (9-11, 21-23). Com exceção do consumo de risco para álcool, todos os demais fatores estiveram presentes, em homens e mulheres, com frequências superiores a $25 \%$. A baixa frequência do consumo de frutas e vegetais foi também verificada no Inquérito Mundial de Saúde conduzido no Brasil em 2003 (22), e na Pesquisa de Orçamento Familiar de 2002/2003 (23). Optou-se por investigar a frequência semanal, ao invés do consumo diário de cinco porções desses alimentos, porque mais de $95 \%$ da população não seguiam o padrão diário preconizado pela OMS (24). Portanto, entendemos que a análise baseada nessas recomendações não iria discriminar a amostra quanto à presença ou ausência desse fator.

O inquérito domiciliar cujos dados utilizamos (13) permitiu ainda, pela primeira vez, a aferição pelo IPAQ do nível de atividade física em um estudo de base populacional envolvendo várias regiões do Brasil. $\mathrm{O}$ uso desse instrumento, padronizado e validado no país (25), amplia a capacidade de comparação com outros estudos, especialmente os internacionais. Hallal et al. (26) avaliaram o nível de atividade física em adultos das cidades de São Paulo (estado de São Paulo) e Pelotas (Rio Grande do Sul) e classificaram cerca de $40 \%$ dos entrevistados como inativos fisicamente (sedentários e irregularmente ativos), coincidindo com a proporção encontrada no nosso estudo. Chama a atenção o fato de esse fator ter sido o mais prevalente em todas as categorias do escore de FRCV. Isso pode se dever, em parte, à falta de orientação da população acerca dos benefícios da atividade física no cotidiano, seja nas atividades domésticas, no deslocamento (ao trabalho ou escola) ou no lazer, e, ainda, à carência de locais adequados e seguros para a realização de atividade física.

A decisão de incluir a circunferência da cintura no escore de aglomeração

TABELA 3. Análise univariada da associação entre aglomeração de fatores de risco cardiovascular modificáveis e variáveis selecionadas em adultos de 30 a 69 anos, Brasil, 2002 a 2003

\begin{tabular}{|c|c|c|c|}
\hline \multirow[b]{2}{*}{ Variável } & \multicolumn{3}{|c|}{ Escore de aglomeração de fatores de risco cardiovascular ${ }^{a}$} \\
\hline & 1 & OR e IC $95 \%$ & 3 ou mais \\
\hline Idade & $1,01(1,00$ a 1,02$)$ & $1,03(1,01$ a 1,03$)$ & $1,04(1,03$ a 1,05$)$ \\
\hline \multicolumn{4}{|l|}{ Sexo } \\
\hline Feminino & 1,00 & 1,00 & 1,00 \\
\hline Masculino & $0,87(0,73$ a 1,05$)$ & $0,76(0,63$ a 0,92$)$ & $0,85(0,70$ a 1,04$)$ \\
\hline \multicolumn{4}{|l|}{ Escolaridade (anos) } \\
\hline 0 a 3 & 1,00 & 1,00 & 1,00 \\
\hline 4 a 7 & $0,79(0,58$ a 1,09$)$ & $0,83(0,59$ a 1,17$)$ & $0,81(0,54$ a 1,18$)$ \\
\hline 8 a 11 & $0,72(0,54$ a 0,96$)$ & $0,53(0,37$ a 0,74$)$ & $0,41(0,30$ a 0,57$)$ \\
\hline$\geq 12$ & $0,54(0,39$ a 0,75$)$ & $0,39(0,27$ a 0,58$)$ & $0,24(0,15$ a 0,36$)$ \\
\hline \multicolumn{4}{|c|}{ Renda familiar (salários mínimos) } \\
\hline$<3$ & 1,00 & 1,00 & 1,00 \\
\hline $3 a<5$ & $0,55(0,41$ a 0,73$)$ & $0,61(0,46$ a 0,81$)$ & $0,49(0,35$ a 0,68$)$ \\
\hline $5 a<10$ & $0,60(0,45$ a 0,79$)$ & $0,52(0,38$ a 0,64$)$ & $0,49(0,36$ a 0,67$)$ \\
\hline$\geq 10$ & $0,49(0,38$ a 0,64$)$ & $0,42(0,32$ a 0,55$)$ & $0,27(0,20$ a 0,38$)$ \\
\hline \multicolumn{4}{|l|}{ Percepção da saúde } \\
\hline Excelente/muito boa & 1,00 & 1,00 & 1,00 \\
\hline Boa & $1,30(1,05$ a 1,61$)$ & $1,69(1,35$ a 2,12) & $1,86(1,41$ a 2,46$)$ \\
\hline Regular & $2,48(1,91$ a 3,22) & 4,62 (3,47 a 6,14) & $6,71(4,94$ a 9,12$)$ \\
\hline Ruim & 3,32 (1,37 a 8,06) & $8,5$ (3,57 a 20,23$)$ & $21,17(9,31$ a 48,15$)$ \\
\hline \multicolumn{4}{|l|}{ Morbidade referida } \\
\hline Diabetes & $2,65(1,48$ a 4,72$)$ & 3,45 (1,92 a 6,22) & $4,52(2,45$ a 8,34$)$ \\
\hline Doença isquêmica & $1,24(0,64$ a 2,41$)$ & $2,51(1,35$ a 4,65) & $2,69(1,44$ a 5,02$)$ \\
\hline
\end{tabular}

Fonte: Inquérito Domiciliar sobre Comportamentos de Risco e Morbidade Referida de Doenças e Agravos Não Transmissíveis, Comprev/INCA/Ministério da Saúde (13).

a Escore de aglomeração de fatores de risco cardiovascular definido como a soma de qualquer combinação entre os seguintes fatores: hipertensão, tabagismo, dieta inadequada, uso excessivo de álcool, inatividade física e obesidade central.

b Razão de chances (odds ratio) e intervalo de confiança de $95 \%$ obtidos por regressão logística multinomial, tendo como categoria de referência a ausência de fatores de risco cardiovascular.

TABELA 4. Análise multivariada da associação entre aglomeração de fatores de risco cardiovascular modificáveis e variáveis selecionadas em adultos de 30 a 69 anos, Brasil, 2002 a 2003

\begin{tabular}{|c|c|c|c|}
\hline \multirow[b]{2}{*}{ Variável } & \multicolumn{3}{|c|}{$\begin{array}{l}\text { Escore de aglomeração de fatores de risco cardiovascular }{ }^{\mathrm{a}} \\
\qquad(n=7256)\end{array}$} \\
\hline & 1 & OR e IC ${ }^{2}$ (C5\% & 3 ou mais \\
\hline Idade & $1,01(1,00$ a 1,02$)$ & $1,02(1,01$ a 1,04$)$ & $1,03(1,02$ a 1,05$)$ \\
\hline \multicolumn{4}{|l|}{ Sexo } \\
\hline Feminino & 1,00 & 1,00 & 1,00 \\
\hline Masculino & $0,95(078$ a 1,14$)$ & $0,90(0,73$ a 1,10$)$ & $1,06(0,85$ a 1,31$)$ \\
\hline \multicolumn{4}{|c|}{ Renda familiar (salários mínimos) } \\
\hline$<3$ & 1,00 & 1,00 & 1,00 \\
\hline $3 a<5$ & $0,58(0,44$ a 0,76$)$ & $0,67(0,51$ a 0,88$)$ & $0,55(0,39$ a 0,76$)$ \\
\hline $5 a<10$ & $0,64(0,48$ a 0,84$)$ & $0,58(0,43$ a 0,78$)$ & $0,57(0,35$ a 0,74$)$ \\
\hline$\geq 10$ & $0,56(0,43$ a 0,73$)$ & $0,54(0,41$ a 0,72$)$ & $0,39(0,27$ a 0,55$)$ \\
\hline \multicolumn{4}{|l|}{ Percepcão da saúde } \\
\hline Excelente/muito boa & 1,00 & 1,00 & 1,00 \\
\hline Boa & $1,24(1,01$ a 1,53$)$ & $1,59(1,27$ a 2,00$)$ & $1,67(1,27$ a 2,20$)$ \\
\hline Regular & $2,21(1,67$ a 2,92$)$ & $3,85(2,85$ a 5,19$)$ & $5,28(3,79$ a 7,36$)$ \\
\hline Ruim & $2,80(1,15$ a 6,78$)$ & $6,13(2,62$ a 14,34$)$ & $14,60(6,45$ a 33,04$)$ \\
\hline
\end{tabular}

Fonte: Inquérito Domiciliar sobre Comportamentos de Risco e Morbidade Referida de Doenças e Agravos Não Transmissíveis, Comprev/INCA/Ministério da Saúde (13).

a Escore de aglomeração de fatores de risco cardiovascular definido como a soma de qualquer combinação entre os seguintes fatores: hipertensão, tabagismo, dieta inadequada, uso excessivo de álcool, inatividade física e obesidade central.

${ }^{b}$ Razão de chances (odds ratio) e intervalo de confiança de $95 \%$ obtidos por regressão logística multinomial, tendo como categoria de referência a ausência de fatores de risco cardiovascular.

deve-se ao reconhecimento de que a obesidade central representa um fator de risco independente do índice de massa corporal (IMC) para diversas doenças crônicas não transmissíveis, sobretudo as cardiovasculares (27), além de ser a única variável aferida na população estudada. Em nível populacional, essa me- 
TABELA 5. Análise multivariada da associação entre aglomeração de fatores de risco cardiovascular modificáveis (sem hipertensão) e variáveis selecionadas em adultos de 30 a 69 anos, Brasil, 2002 a 2003

\begin{tabular}{|c|c|c|c|}
\hline \multirow[b]{2}{*}{ Variável } & \multicolumn{3}{|c|}{$\begin{array}{l}\text { Escore de aglomeração de fatores de risco cardiovascular }{ }^{\mathrm{a}} \\
\qquad(n=7528)\end{array}$} \\
\hline & 1 & OR e IC $\stackrel{2}{\mathrm{I}} 95 \%$ & 3 ou mais \\
\hline Idade & $1,00(0,99$ a 1,01$)$ & $1,00(1,00$ a 1,02$)$ & $1,00(0,98$ a 1,01$)$ \\
\hline \multicolumn{4}{|l|}{ Sexo } \\
\hline Feminino & 1,00 & 1,00 & 1,00 \\
\hline Masculino & $0,92(0,76$ a 1,11$)$ & $1,06(0,86$ a 1,32$)$ & $1,32(1,00$ a 1,76$)$ \\
\hline \multicolumn{4}{|c|}{ Renda familiar (salários mínimos) } \\
\hline$<3$ & 1,00 & 1,00 & 1,00 \\
\hline $3 a<5$ & $0,81(0,57$ a 1,15$)$ & $0,66(0,46$ a 0,95$)$ & $0,53(0,34$ a 0,83$)$ \\
\hline $5 a<10$ & $0,66(0,47$ a 0,92$)$ & $0,55(0,38$ a 0,79$)$ & $0,49(0,32$ a 0,75$)$ \\
\hline$>10$ & $0,60(0,45$ a 0,79$)$ & $0,52(0,38$ a 0,72$)$ & $0,29(0,18$ a 0,45$)$ \\
\hline \multicolumn{4}{|l|}{ Percepção da saúde } \\
\hline Excelente/muito boa & 1,00 & 1,00 & 1,00 \\
\hline Boa & $1,35(1,08$ a 1,68$)$ & $1,47(1,13$ a 1,90$)$ & $1,88(1,30$ a 2,71$)$ \\
\hline Regular & $1,82(1,38$ a 2,42$)$ & $2,55(1,86$ a 3,50$)$ & $2,49(1,60$ a 3,87$)$ \\
\hline Ruim & $2,28(1,15$ a 4,54$)$ & $3,76(1,82$ a 7,75$)$ & $9,27(4,10$ a 20,96$)$ \\
\hline \multicolumn{4}{|l|}{ Morbidade referida } \\
\hline Hipertensão & $1,21(0,95$ a 1,56$)$ & $1,67(1,26$ a 2,22$)$ & $1,58(1,12$ a 2,21$)$ \\
\hline Diabetes & $0,86(0,53$ a 1,39$)$ & $0,76(0,46$ a 1,27$)$ & $0,80(0,48$ a 1,35$)$ \\
\hline Doença isquêmica & $1,03(0,64$ a 1,67$)$ & $0,63(0,37$ a 1,08$)$ & $0,50(0,26$ a 0,97$)$ \\
\hline
\end{tabular}

Fonte: Inquérito Domiciliar sobre Comportamentos de Risco e Morbidade Referida de Doenças e Agravos Não Transmissíveis, Comprev/INCA/Ministério da Saúde (13).

${ }^{a}$ Escore de aglomeração de fatores de risco cardiovascular definido como a soma de qualquer combinação entre os seguintes fatores: hipertensão, tabagismo, dieta inadequada, uso excessivo de álcool, inatividade física e obesidade central.

${ }^{b}$ Razão de chances (odds ratio) e intervalo de confiança de $95 \%$ obtidos por regressão logística multinomial, tendo como categoria de referência a ausência de fatores de risco cardiovascular.

dida antropométrica tem a vantagem de ser prática e de fácil utilização. Além disso, possibilita identificar níveis de intervenção para promoção de saúde.

A prevalência de obesidade central foi alta (30\%). A utilização do ponto de corte de $88 \mathrm{~cm}$ para mulheres e 102 para homens, considerado parâmetro para um nível de intervenção que inclui medidas de prevenção secundárias, indica ser aconselhável o acompanhamento dos indivíduos por profissionais da saúde a fim de reduzir peso e consequente diminuição do risco $(17,28)$. A combinação entre envelhecimento populacional e aumento da urbanização causa modificações nos modos de vida que propiciam o sedentarismo, a adoção de hábitos alimentares menos saudáveis e a obesidade, entre outros, criando condições favoráveis ao desenvolvimento das doenças cardiovasculares. Neste estudo, realizado em metrópoles brasileiras, cerca da metade dos entrevistados apresentou 2 ou mais dos FRCV investigados (inatividade física, dieta inadequada, obesidade central, tabagismo, consumo de risco de álcool e hipertensão). A maior frequência de fatores de risco com o aumento da idade e a maior aglomeração de fatores de risco em homens residentes em áreas urbanas são conceitos bastante difundidos na literatura $(9-11,29-32)$. No entanto, não encontramos diferenças significativas quanto ao número de fatores por gênero.

Menor aglomeração de fatores de risco foi observada entre aqueles com maior renda e escolaridade, melhor autoavaliação da saúde e entre os indivíduos com relato negativo de DIC e diabetes, corroborando resultados obtidos em outros estudos (3, 5, 7, 12, 29-32). A relação entre maior educação ou renda e menor carga de fatores de risco cardiovascular pode sugerir maior acesso às informações e aos serviços de saúde, tendo como resultado a prática de comportamentos saudáveis. Porém, a natureza dessa relação é complexa, varia ao longo da vida e não pode ser captada em estudos transversais.

No Brasil, estudos de base populacional realizados nas regiões Sul (11) e Sudeste (9) descreveram uma frequência menor de aglomeração de 2 ou mais fatores, 39 e $31 \%$ respectivamente. Já em Salvador, região Nordeste do país, cerca de $70 \%$ dos entrevistados apresentaram pelo menos 2 fatores investigados (10).

Os estudos nacionais e internacionais sobre aglomeração de fatores de risco apresentam grandes variações nos conjuntos de fatores de risco analisados, nas técnicas de medidas e nas amostragens, o que dificulta a comparabilidade dos mesmos. Cabe ressaltar que a hipercolesterolemia integra a maioria dos estudos sobre aglomeração de FRCV. Entretanto, esse fator não foi incluído neste estudo devido ao elevado número de perdas desta informação nas entrevistas com adultos. Além disso, muitas investigações realizadas nas últimas décadas categorizam indivíduos e população em baixo, médio ou alto risco. Assim, não foi possível fazer uma comparação direta com os resultados encontrados.

Nossos resultados mostraram que a probabilidade de referir DIC e diabetes foi maior na presença de maior aglomeração de FRCV, coincidindo com resultados de inúmeros estudos que destacam maior incidência de eventos cardiovasculares e aumento da mortalidade por qualquer causa sempre que um fator de risco é adicionado $(4,32)$.

A análise do escore de aglomeração de FRCV com e sem a presença de hipertensão mostrou associação significativa com idade apenas quando a hipertensão foi incluída. A sua adição no escore, além de resultar em maior impacto negativo sobre a percepção da saúde, implicou no aumento da associação com DIC e diabetes. A influência da HAS na associação com idade é esperada, já que este fator está claramente relacionado ao aumento da idade, independentemente da aglomeração com outros fatores de risco (33). Além disso, a maior associação com os desfechos estudados (DIC e diabetes) coincide com resultados de investigações prévias mostrando a tendência de a hipertensão ocorrer concomitantemente com outras desordens metabólicas, com aumento do risco cardiovascular (34). Esses resultados reforçam a necessidade do diagnóstico e tratamento precoces da hipertensão, assim como de uma abordagem integral do perfil de risco dessa população. A prevalência isolada da hipertensão (27\%) foi similar àquela estimada em outros estudos de base populacional realizados no Brasil, considerando as diferenças nas medidas para classificação da população, na distribuição etária e nível social dos grupos investigados $(9,10)$.

A autoavaliação da saúde mostrou forte associação com o perfil de risco cardiovascular, com gradiente positivo de associação entre número de fatores e 
avaliação da saúde e, especialmente, com pior percepção da saúde. $\mathrm{O}$ ajuste para possíveis fatores de confusão, como idade, sexo, escolaridade e renda, e a presença de doenças, como diabetes e DIC, não alteraram a direção dessa associação, corroborando a sua importância como marcador de saúde cardiovascular. Este resultado concorda com os achados publicados recentemente da análise de dois inquéritos de base populacional, um nos Estados Unidos e outro na Suécia (9). Cabe destacar que a percepção da saúde sofre influência de diferentes processos e condições, que incluem suporte social, acesso aos serviços de saúde, privação e depressão, entre outros $(7,8,12,35)$ não analisados neste estudo.

A investigação da coexistência de FRCV é importante, sobretudo, devido à interação entre os fatores e sua implicação direta na gênese, progressão e ocorrência de eventos cardiovasculares futuros $(3,4)$. No entanto, estudos recentes têm questionado o efeito da combinação entre FRCV, argumentando que os fatores analisados isoladamente não têm a mesma importância para predizer o risco cardiovascular, o que significa que, nem sempre, as aglomerações resultarão em efeito sinérgico (36). Apesar da utilização de um escore simplificado, que não faz distinção entre os componentes, o presente estudo permitiu traçar o perfil de saúde cardiovascular da população brasileira de forma abrangente, com implicações importantes para a promoção de saúde no nível populacional.

Embora tenha sido realizado em 16 capitais, incluindo as maiores do país, o inquérito cujos dados utilizamos não representa toda a população brasileira, e as características da amostra não permitem desagregar os dados por região. Por ser um estudo transversal, não é possível fazer inferências causais ou temporais acerca da associação entre variáveis dependentes e independentes (37). Apesar dos inúmeros estudos que indicam uma boa confiabilidade das informações autorreferidas $(18-20,38,39)$, sabe-se que estas podem sofrer influência do acesso aos serviços de saúde, viés de memória e outros (40).
Em resumo, a elevada prevalência de múltiplos fatores de risco para doenças cardiovasculares que relatamos reforça a importância de políticas públicas voltadas para a redução desses fatores. Além disso, os resultados dessas intervenções devem ser monitorados por inquéritos periódicos de base populacional, capazes de detectar mudanças no perfil de risco, identificar subgrupos expostos a maior risco, nortear e avaliar o impacto de estratégias de prevenção. A associação direta e significativa entre autoavaliação da saúde e perfil de risco cardiovascular em adultos reforça a aplicabilidade desse indicador em inquéritos populacionais como marcador de saúde e aponta a necessidade de investigações futuras acerca de outras variáveis que influenciem essa relação.

Agradecimentos. Ao COMPREV/ Instituto Nacional do Câncer e Secretaria de Vigilância à Saúde do Ministério da Saúde pelo acesso ao banco de dados. SMB e VMAP são pesquisadoras do CNPq (n³00908/95 e 300159/99-4).

\section{REFERÊNCIAS}

1. Brasil. Ministério da Saúde. Secretaria de Vigilância em Saúde. Indicadores de mortalidade. Brasil: Ministério da Saúde; 2006. Disponível em: http://tabnet.datasus.gov.br/ cgi/tabcgi.exe?idb2006/c04.def. Acessado em 15 de dezembro de 2007.

2. World Health Organization. The world health report 2002-Reducing risks, promoting health life. Disponível em: http://www.who. int/whr/2002/media_centre/en/index.html. Acessado em 14 de novembro de 2005.

3. Ebrahim S, Montaner D, Lawlor DA. Clustering of risk factors and social class in childhood and adulthood in British women's heart and health study: cross sectional analysis. BMJ. 2004;328(7444):861.

4. Ezzati M, Hoorn SV, Rodgers A, Lopez AD, Mathers, CD, Murray CJ, et al. Estimates of global and regional potential health gains from reducing multiple major risk factors. Lancet. 2003;362(9380):271-80.

5. Emmelin M, Weinehall L, Stenlund H, Wall S, Dahlgren L. To be seen, confirmed and involved-a ten year follow-up of perceived health and cardiovascular risk factors in a Swedish community intervention programme. BMC Public Health. 2007;7:190.

6. Yusuf S, Reddy S, Ounpuu S, Anand S. Global burden of cardiovascular diseases: Part II: variations in cardiovascular disease by specifics ethnic groups and geographic regions and prevention strategies. Circulation. 2001; 104(23):2855-64.

7. Molarius A, Berglund K, Eriksson C, Lambe M, Norstrom E, Eriksson HG, et al. Socio- economic conditions, lifestyle factors, and self-rated health among men and women in Sweden. Eur J Public Health. 2007;17(2): $125-33$.

8. Centers for Disease Control and Prevention (CDC). Self-rated fair or poor health among adults with diabetes-United States, 19962005. MMWR Morb Mortal Wkly Rep. 2006; 55(45):1224-7.

9. Barreto SM, Passos VM, Firmo JO, Guerra HL, Vidigal PG, Lima-Costa MF. Hypertension and clustering of cardiovascular risk factors in a community in Southeast Brazil-the Bambuí Health and Ageing Study. Arq Bras Cardiol. 2001;77(6):576-81.

10. Lessa I, Araújo MJ, Magalhães L, Almeida Filho N, Aquino E, Costa MC. Simultaneidade de fatores de risco cardiovascular modificáveis na população adulta de Salvador (BA), Brasil. Rev Panam Salud Publica. 2004;16(2): 131-7.

11. Duncan BB, Schmidt MI, Polanczyk CA, Homrich CS, Rosa RS, Achutti AC. Fatores de risco para doenças não transmissíveis em área metropolitana na região sul do Brasil. Prevalência e simultaneidade. Rev Saude Publica. 1993;27(1):143-8.

12. Emmelin $M$, Nafziger NA, Stenlund $H$, Weinehall L, Wall S. Cardiovascular risk factor burden has a stronger association with self-rated poor health in adults in the US than in Sweden, especially for the lower educated. Scand J Public Health. 2006;34(2):140-9.

13. Brasil. Ministério da Saúde. Instituto Nacional de Câncer. Inquérito domiciliar sobre com- portamentos de risco e morbidade referida de doenças e agravos não transmissíveis: Brasil, 15 capitais e Distrito Federal, 2002-2003. Rio de Janeiro: INCA; 2004.

14. World Health Organization. Cardiovascular disease: CVD Prevention and control: missed opportunities. Disponível em: http://www. who.int/cardiovascular_diseases/prevention control/en/. Acessado em 20 de julho de 2006.

15. Centers for Disease Control and Prevention (CDC). 2000. Behavioral Risk Factor Surveillance System Spanish Questionnaire. Disponível em: http://www.cdc.gov/brfss/ questionnaires/pdf-ques/q2000cspan.pdf. Acessado em agosto de 2006.

16. World Health Organization. International Physical Activity Questionnaires (IPAQ) Scoring Protocol. Genebra: WHO; 2001. Disponível em: http://www.ipaq.ki.se/scoring. $\mathrm{htm}$. Acessado em 8 de agosto de 2006.

17. World Health Organization. Physical status: the use and interpretation of anthropometric indicators of nutritional status. Genebra: $\mathrm{WHO}$; 1995. (WHO Technical Report Series 854).

18. Lima-Costa MF, Peixoto SV, Firmo JOA. Validade da hipertensão arterial auto-referida e seus determinantes (projeto Bambuí). Rev Saude Publica. 2004;38(5):637-42.

19. Fonseca MJM, Faerstein E, Chor D, Lopes CS. Validade de peso e estatura informados e índice de massa corporal: estudo pró-saúde. Rev Saude Publica. 2004;38(3):392-8.

20. Chor D, Faerstein E, Alves MG, de Souza Lopes C. How reproducible is self-reported information on exposure to smoking, drink- 
ing, and dietary patterns? Evidence among Brazilian adults in the Pró-Saúde Study. Sao Paulo Med J. 2003;121(2):63-6.

21. Schuit AJ, van Loon AJ, Tijhuis M, Ocké M. Clustering of lifestyle risk factors in a general adult population. Prev Med. 2002;35(3):219-24.

22. Jaime PC, Monteiro CA. Fruit and vegetables intake by Brazilian adults, 2003. Cad Saude Publica. 2005;21 Supl 1:S19-24.

23. Instituto Brasileiro de Geografia e Estatística. Pesquisa de orçamentos familiares 2002-2003. Análise da disponibilidade domiciliar de alimentos e do estado nutricional no Brasil. Rio de Janeiro: IBGE; 2004. Notas Técnicas. Disponível em: http://www.ibge.gov.br/home/ estatistica/populacao/condicaodevida/pof/ 2002analise/notatecnica.pdf. Acessado em maio de 2009.

24. World Health Organization. Global Strategy on Diet, Physical Activity and Health. Obesity and overweight. Genebra: WHO; 2004. Disponível em: http://www.who.int/dietphysical activity/publications/facts/obesity/en/. Acessado em 8 de agosto de 2006.

25. Matsudo S, Araújo T, Matsudo V, Andrade D, Andrade E, Oliveira $C$, et al. Questionário Internacional de Atividade Física (IPAQ): estudo de validade e reprodutibilidade no Brasil. Rev Bras Ativ Fis Saude. 2001;6(2):5-12.

26. Hallal PC, Matsudo SM, Matsudo VKR, Araújo TL, Andrade DR, Bertoldi AD. Physical activity in adults from two Brazilian areas: similarities and differences. Cad Saude $\mathrm{Pu}$ blica. 2005;21(2):573-80.

27. Gus M, Fuchs SC, Moreira LB, Moraes RS, Wiehe M, Silva AF, et al. Association between different measurements of obesity and the incidence of hypertension. Am J Hypertens. 2004;17(1):50-3.

28. Lean MEJ, Han TS, Morrison CE. Waist circumference as a measure for indicating need for weight management. BMJ. 1995;311(6998): 158-61.

29. Gu D, Gupta A, Muntner P, Hu S, Duan X, Chen J, et al. Prevalence of cardiovascular disease risk factor clustering among the adult population of China: results from the International Collaborative Study of Cardiovascular Disease in Asia (InterAsia). Circulation. 2005; 112(5):658-65.

30. Greenlund KJ, Zheng ZJ, Keenan NL, Giles WH, Casper ML, Mensah GA, et al. Trends in self-reported multiple cardiovascular disease risk factors among adults in the United States, 1991-1999. Arch Intern Med. 2004;164(2):181-8.

31. NHANES 1999-2000 addendum to the NHANES III analytic guidelines. Disponível em: http://www.cdc.gov/nchs/data/nhanes/ guidelines1.pdf. Acessado em $1^{\circ}$ de setembro de 2004.

32. Stamler J, Stamler R, Neaton JD, Wentworth D, Daviglus ML, Garside D, et al. Low riskfactor profile and long-term cardiovascular and noncardiovascular mortality and life expectancy: findings for 5 large cohorts of young adult and middle-aged men and women. JAMA. 1999;282(21):2012-8.

33. Ramos R, Marrugat J, Basagaña $X$, Sala J, Masiá R, Elosua R, et al. The role of age in cardiovascular risk factor clustering in nondiabetic population free of coronary heart disease. Eur J Epidemiol. 2004;19(4):299-304.
34. Kannel WB. Risk stratification in hypertension new insights from the Framingham Study. Am J Hypertens. 2000;13(1 Pt 2):3S-10S.

35. Manderbacka K, Lundberg O, Martikainen P. Do risk factors and health behaviors contribute to self-ratings of health? Soc Sci Med. 1999;48(12):1713-20.

36. Wang J, Ruotsalainen S, Moilanen L, Lepisto P, Laakso M, Kuusisto J. The metabolic syndrome predicts cardiovascular mortality: a 13-year follow-up study in elderly nondiabetic Finns. Eur Heart J. 2007;28(7):857-64.

37. Friis RH, Sellers TA. Study designs. Em: Epidemiology for public health practice. Gaithersburg: Aspen; 1999. Pp. 87-229.

38. Martin LM, Leff M, Calonge N, Garret C, Nelson DE. Validation of self-reported chronic conditions an health services in a managed care population. Am J Prev Med. 2000;18(3): 215-8.

39. Colditz GA, Martin P, Stampfer MJ, Willet WC, Sampson L, Rosner B, et al. Validation of questionnaire information on risk factors and diseases outcomes in a prospective cohort study of women. Am J Epidemiol. 1986; 123(5):894-900.

40. Lima-Costa MF, Peixoto SV, Firmo JOA, Uchoa E. Validade do diabetes auto-referido e seus determinantes: evidências do projeto Bambuí. Rev Saude Pub. 2007;41(6):947-53.

Manuscrito recebido em 16 de abril de 2008. Aceito em versão revisada em 25 de julho de 2008.
ABSTRACT

\section{Cardiovascular risk} profile and health self-evaluation in Brazil: a population-based study

Objectives. To estimate the prevalence and clustering of cardiovascular risk factors (hypertension, smoking, physical inactivity, excessive use of alcohol, inadequate diet, and central obesity) and investigate the association of these factors with health selfevaluation after adjustment for sociodemographic features and clinical diagnosis of diabetes and ischemic heart disease.

Methods. We studied 9211 Brazilians with age between 30 and 69 years, residents of 16 state capitals, included in a cross-sectional population-based study carried out by the Ministry of Health (Inquérito Domiciliar sobre Comportamentos de Risco e Morbidade Referida de Doenças e Agravos Não Transmissiveis, 2002-2003). The response variable was the score of cardiovascular risk factor clustering, which ranged from zero to 3: no exposure or exposure to 1,2, and 3 or more risk factors. Data were analyzed using multinomial logistic regression, having as reference the category of zero risk factors, with and without hypertension.

Results. The prevalence of all risk factors except alcohol consumption was higher than $25 \%$. The presence of two or more risk factors occurred in $47.49 \%$; $17.91 \%$ of the participants presented zero risk factors. The clustering of risk factors was inversely associated with income and schooling and higher in the age group between 50 and 59 years, without significant differences between sexes. Health self-evaluation was worse in participants with more risk factors. The inclusion of hypertension in the score resulted in a worsening of the risk profile with increase in age and in a significant association with reported ischemic heart disease and diabetes.

Conclusions. The high prevalence of cardiovascular risk factors in Brazilian adults underscores the importance of policies aimed at reducing these factors. The association between worse self-perception of health and a higher number of cardiovascular risk factors calls attention to the importance, broadness and complexity of this subjective health marker in population surveys and suggests that self-perception of poor health is not sufficient to encourage healthy attitudes.

Key words Cardiovascular diseases, risk factors, self-concept, Brazil. 